\title{
Do8Now: AN INTELLIGENT MOBILE PlatForm FOR Time MANAgEMENT USING SOCIAL COMPUTING AND MACHINE LEARNING
}

\author{
Ruichu (Eric) Xia ${ }^{1}$, Yu Sun ${ }^{2}$, Fangyan Zhang ${ }^{3}$ \\ ${ }^{1}$ Santa Margarita Catholic High School, Rancho Santa Margarita, \\ CA 92688, USA \\ ${ }^{2}$ California State Polytechnic University, Pomona, CA, 91768, USA \\ ${ }^{3}$ ASML, San Jose, CA, 95131, USA
}

\begin{abstract}
Many people today suffer from the negative effects of procrastination and poor time management which includes lower productivity, missing opportunities, lower self-esteem and increased levels of guilt, stress, frustration, and anxiety. Although people can often recognize their tendency to procrastinate and the need to change this bad habit, the majority of them still do not take meaningful actions to prevent themselves from procrastinating. To help people fix this problem, we created a goal tracking mobile application called iProgress that aims to assist and motivate people to better manage their time by allowing them to create short-term and long-term goals that they want to achieve, and encouraging them to complete those goals through a rank/reward system that provides them with the opportunity to compete with other users by completing more goals.
\end{abstract}

\section{KEYWORDS}

Procrastination, iProgreass, flutter, iOS, Android

\section{INTRODUCTION}

Procrastination is the intentional avoidance or delay of completing a certain task that is often stressful and unpleasant but also important. Although being a common experience for most people and not a mental health diagnosis by itself, procrastination can cause many negative emotions such as guilt, frustration, and anxiety, and is associated with some serious mental disorders such as attention-deficit hyperactivity disorder (ADHD), depression, and anxiety disorders according to various psychological research studies [1] [2][3]. In one of the first studies conducted on the effects of procrastination published in 1997, the researchers at Case Western Reserve University found that among the participants, students from the university, those who procrastinated generally displayed poorer performance at school, earned lower grades, and reported higher levels of stress than those who did not procrastinate [13]. Procrastination is also very prevalent today as shown in another study conducted in 2004, from which about $70 \%$ of university students reported that they would consider themselves to be procrastinators. Interestingly, the tendency to procrastinate is not only limited to humans. According to one study conducted on the behavioral patterns of pigeons, the researchers have found pigeons also display clear evidence of procrastination [4]. While most people may not experience some of the most 
severe impacts of procrastinating and may sometimes even benefit from it as procrastination can act as a coping mechanism for stressful situations and mood regulations, chronic procrastinators can have their lives persistently disrupted and their success severely limited due to procrastination. To individuals that have the issue of chronic procrastination, the effect may include poor performance at school or work, financial difficulties, increasing levels of stress and anxiety, and poor physical health resulted from lack of exercise and nutrition.

In order to address the problem of procrastination, researchers have been trying to determine the causes of such behavior. Although earlier research has attributed procrastination to an individual's lack of self-regulation and time management skills, recent studies suggest another factor at play that is more important than the other factors: the emotional component of procrastination [5][6]. Studies have shown that people choose to delay or entirely avoid doing certain tasks despite knowing full well the deadline and the consequences of not completing the tasks because they tend to seek the immediate emotional improvements associated with delaying the task at hand. So instead of completing the task way ahead of the deadline, people who tend to procrastinate would choose to put it off for some time because they have the false belief that they will be better and more emotionally equipped at finishing the task in the future. Therefore, along with the lack of self-regulation, the immediate pleasure-seeking temptation causes people to procrastinate. There are some useful intervention methods that reduce procrastination [11][12]. This includes setting up a schedule for the deadlines, breaking down the goal into smaller tasks, and practice to be mindful of certain negative feelings associated with doing the task. Some of these techniques are certainly very helpful to people who tend to procrastinate. For example, setting up a timer for when everything needs to be done is a simple but also very effective way of keeping people on track. However, most of these techniques by themselves cannot actually effectively prevent people from procrastinating. While a simple timer might help a person be aware of what needs to be done before a certain time, the emotional aspect of the problem remains unanswered. Breaking down tasks into smaller pieces is certainly a good technique, but without any external demands and requirements of the procrastinator, it would still be difficult for that person to commit to finishing the entire task without being distracted by temporary pleasure temptations.

In this project, we aim to address the problem of procrastination by combining all the useful strategies in dealing with procrastination into a mobile application called iProgress. Although iProgress is also a habit/goal tracking app that is common on the app market, it has several unique features that can effectively reduce people's tendency to procrastinate, making it overall a better productivity tool than most of the other existing apps. First, an entirely customizable schedule is put in place for the users to set and keep track of their daily tasks and long-term goals. Second, by allowing users to consciously point out the benefit of not putting off a certain goal, the app targets the user's emotions and reward seeking behaviour, encouraging them to avoid temporary distractions. And lastly, inspired by various rank systems in video games, we added a similar rank system to the app that will further motivate its users to complete more goals on time as they compete with each other by completing more goals without procrastinating. Therefore, we believe that with the efficient methods incorporated into the app, iProgress can help people to effectively deal with the problem of procrastination and form a better and more productive lifestyle by developing good work ethics and more healthy habits.

To prove our results, we recruited 20 participants who are interested in using the app and conducted a close interview with each of the participants both before and after 30 days of using the app. Through the interview process, we were able to compare the participant's initial experience in procrastination with their experience after using the app for some time. Then we analyzed the changes in their experiences and determined whether or not the app has an overall positive effect on the participants in helping them avoid procrastination. Moreover, we also 
collected evaluation of the app from the participants as well as any suggestions for potential changes for the app in the future.

The rest of the paper is organized as follows: Section 2 displays the details of the challenges we had in designing the app and implementing the methods and functions; Section 3 gives the details of our solutions regarding each challenge we faced as mentioned in Section 2; Following that, Section 4 presents the relevant details about the user experiences and evaluations; Section 5 lists out some of the related applications on the market, followed by Section 6 which provides the conclusion remarks as well as an overview on the potential future improvements of the application.

\section{Challenges}

Building a mobile application for the first time with a new programming language is not an easy task. Throughout the course of the development, we ran into several challenges that needed overcoming. Here is a brief overview of some of the most difficult challenges that we faced when developing this app.

\subsection{Challenge 1: Coming up with a Solution that Addresses All Aspects of the Problem is Not an Easy Task.}

After doing some research on the issue of procrastination, we noticed that there are many different aspects of the problem that needed to be taken into consideration when we were designing the app. We found that there is no one single method that can effectively address the problem of procrastination. For example, a schedule system that allows users to set and complete goals is not sufficient as the only source of motivation to keep people on track. Therefore, coming up with a comprehensive solution that addresses both the time management aspect and the emotional aspect of procrastination was critical to the effectiveness and the success of the app. Moreover, the details of the rank system also needed to be carefully designed as we wanted to ensure that the system is both fair and rewarding.

\subsection{Challenge 2: Implementing the Design Ideas can be Hard Because the Rank System Requires a Database Server.}

Unlike other goal tracking apps, our design of the app includes a rank system that requires us to have a functional database server to store all the necessary information of the users including points, completed goals, rank position, and username, etc. Without a server, the app would not offer the opportunity for the users to compete with each other, which is an important motivation factor in reducing the tendency of procrastination. Besides implementing a functional server, other design ideas such as separating long-term and short-term goals, implementing a remind/notification system, and making the motivational statement associated with each goal set by the user useful in targeting the emotional aspect of procrastination were all challenges that needed us to address throughout the course of the development.

\subsection{Challenge 3: Building a Cool User Interface and Adding New Functions and Modules are Necessary in Attracting More Users.}

After finishing implementing the basic functions of the app, we began to focus all our attention on building a beautiful user interface with smooth transitions from modules to modules. We knew that having an UI that is both easy to view and easy to understand is crucial in attracting new users as well as keeping the current user base because no one wants to use an app every day that 
is hard to look at and way too complicated to understand. However, connecting different modules and building a cool user interface with flutter for the first time was not easy. Since every page's design had to be written in code, building a good UI required us to learn the new language more thoroughly before knowing how to format each page, connecting them together, and making sure that the app runs smoothly on the user device.

\section{Solution}

The goal of our application is to help the users reduce their tendency to procrastinate. An overview of the app is shown in the diagram below. To use the app, the user is first asked to create an account by providing a username, email and password. Once an account has been created, a new user id will be generated and all the user's information will be initialized in the firebase server, which is the server that we use to store the relevant user information. Then the user can login to his/her account and gain access to the main functions of the app. As seen from the diagram, the app consists of three main functions.

\subsection{Overview of iProgress System}

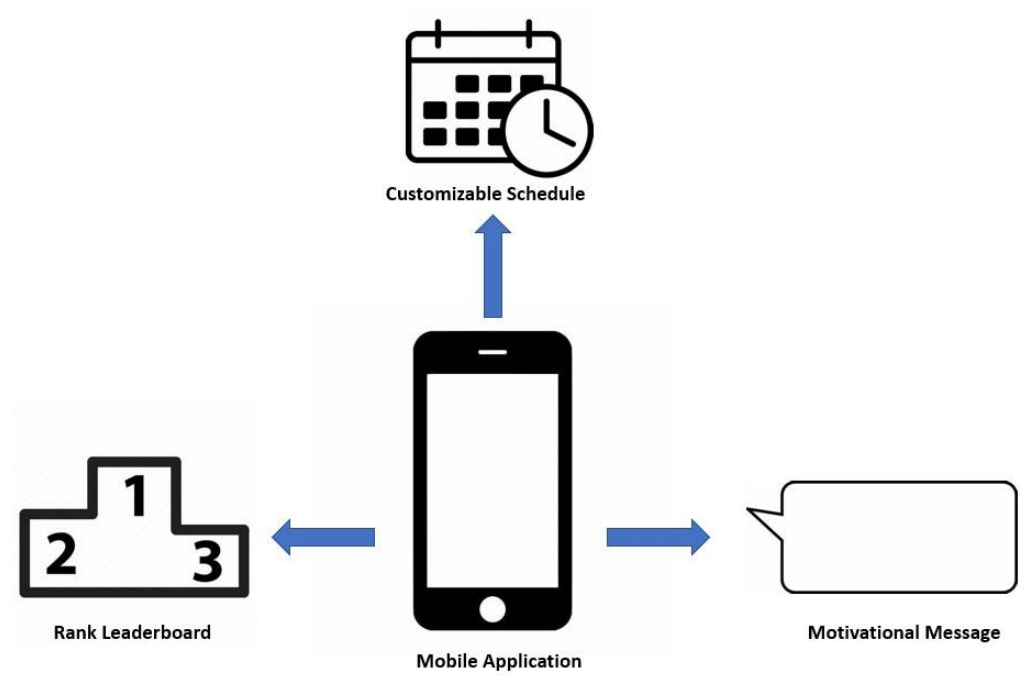

Figure 1: Overview of iProgress system

It allows the user to create a list of small goals and one big goal and helps them to keep track of those goals. This function aims to solve one aspect of procrastination, which is one's inability to efficiently manage schedule and time. The goals are clearly displayed on the homepage of the app with a big goal on top and a list of small goals at the bottom. A check box can be found next to each small task that the user has set to complete. The user has the ability to customize the schedule by adding, removing, and completing goals. Once a small goal/task is completed, the check box will automatically be checked that tells the user that the task has already been completed. When adding new small goals or changing the big goal, the user has the option to either choose from a list of predefined goals on the add goal page or customize a new goal by pressing the customize button on the top of the page. To customize a goal, the user can simply input the name and the perceived difficulty of the goal. After clicking confirm, the user will then be asked to set a reminder time for the goal and also provide a brief statement to why it is necessary to complete the goal on time. Then hit confirm again, the user will return to the homepage and the goal will be changed or added to the list. 
When entering each goal, the user is asked to offer a reason why he/she must complete that goal on time. This message is then displayed as a notification on the user's device at the specific reminder time that the user has set for the goal. By requiring the user to consciously give a reason to not procrastinate and reminding the user of that reason, the app aims to address the emotional aspect of procrastination as the user is reminded that he/she will ultimately feel better if the goal is completed on time.

The rank system is the last major function of the app. To the left of the homepage is the social page where the rankings of all users of the app are located. Inspired by common rank systems in video games, the rank system of this app consists of six different divisions ranging from bronze to master and each division has four tiers. Ranks are represented by different rank badges with different colors that are located on the left side of each user. In order to earn points, the user must complete goals on time without procrastinating. Points are rewarded based on the difficulty of the goal and more points are rewarded for long-term goals than small goals. We hope that with the incorporation of a rank system, the users will have more incentives to keep themselves on track of their work and schedule and avoid being distracted by things that will delay the completion of the goals.

\subsection{The Implementation of iProgress System}

To implement the app, we used flutter, an open-source UI software development kit made by Google [6]. Since flutter allows developers to write apps for both IOS and Android using the same language and code, we were able to write everything once in Android Studio and successfully published the app to both iTunes and Google Play store. For the server we used a firebase real-time database, which is a cloud database that stores and syncs data across all clients in real time. Using the firebase database ultimately helped us overcome the challenge of implementing more complicated functions into the app such as the rank system that required a cloud server that can store and distribute information across different devices [7]. Another challenge was building the user interface after we finished implementing the basic functions of the app. To make sure that we have a clean and smooth UI for the app, we built widgets and put them together using flutter material design, which has a bunch of tools for app developers to decorate their apps. The adaptability of the UI also creates a consistent user experience not only across different platforms, but also different devices. Moreover, because flutter supports both IOS and Android, it is very easy for us to make changes to the code and update the app in stores, which means that we can constantly add new functions and make improvements to the app. There were several new features that were added to the app after the initial publication, including an achievement tab that allows the user to see all of his past completed big goals, a streak system that keeps track of how many days in a row a person has been completing goals, and an user details page that displays the points, streak, and the last achievement of the user. 


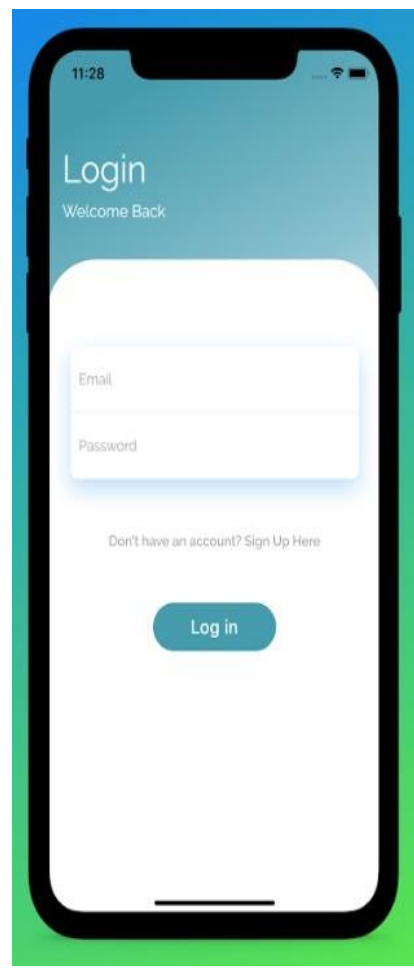

(a)

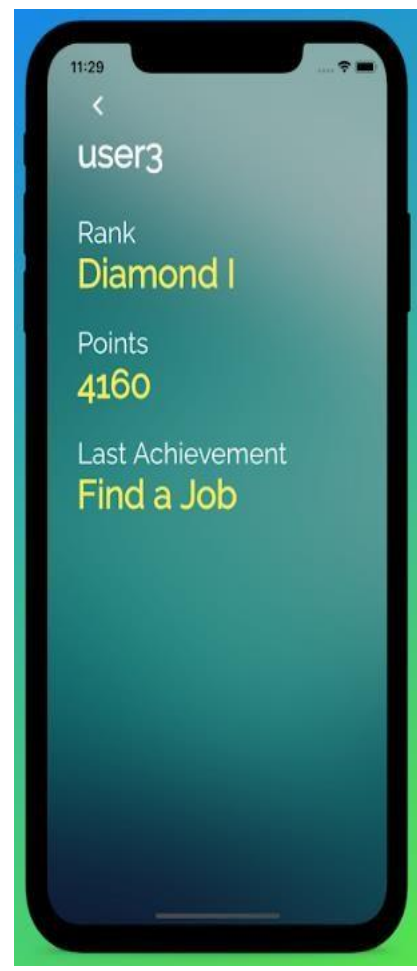

(d)

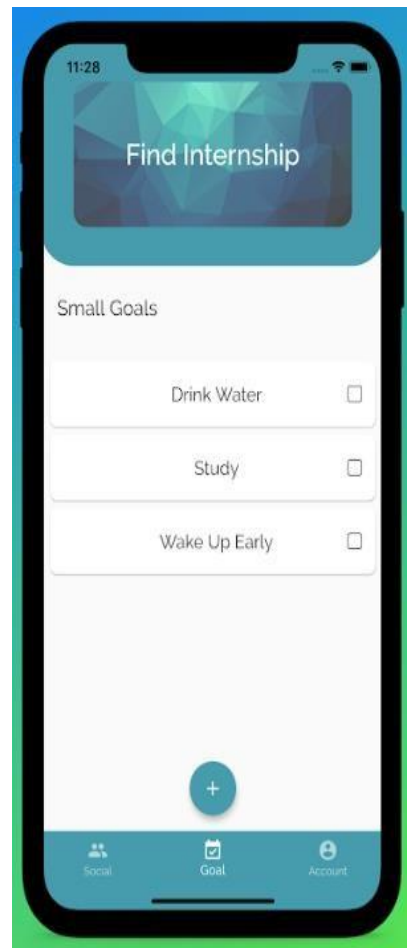

(b)

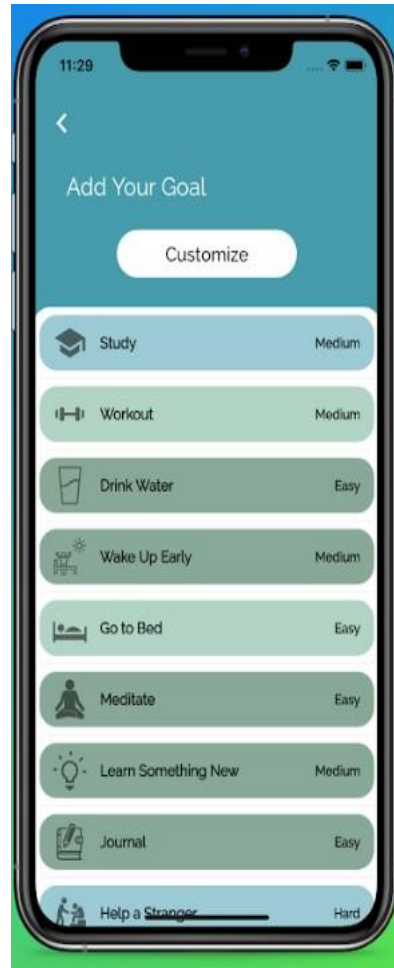

(e)

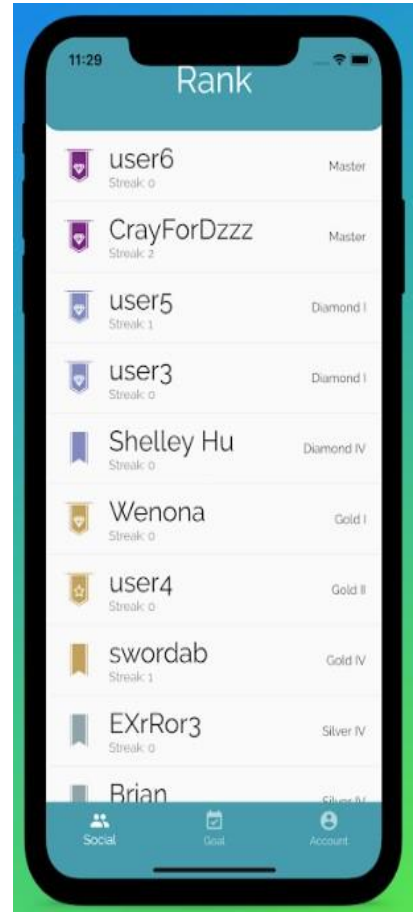

(c)

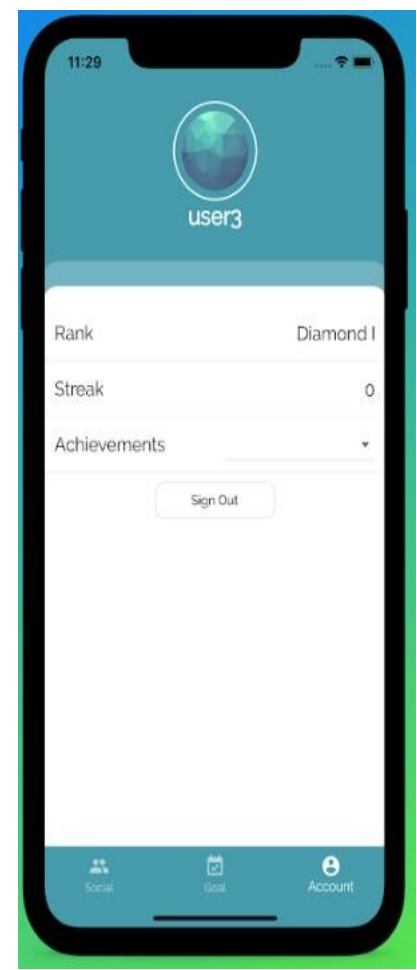

(f)

Figure 2: Interface of iProgress system (a) Log in (b) set a target (c) overall ranks (d) user details (e) summary of a user's goal (f) user rank 


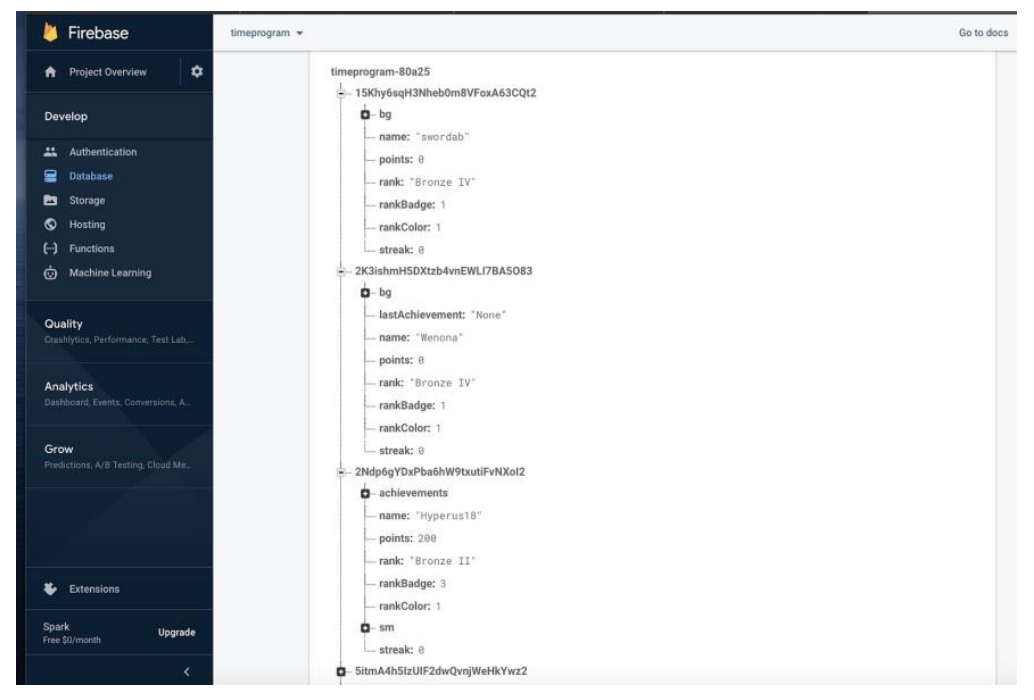

Figure 3: Firebase of iProgress system

\section{EXPERIMENT}

To evaluate the effectiveness of our approach in helping people to deal with procrastination, we collected user evaluation and feedback from 20 participants who used the app for 30 days in the form of close interviews both before and after they began using the app.

In order to determine if the app did actually help reduce people's tendency to procrastinate, the first thing that we did was to compare the initial experience of the participants in dealing with procrastination with their experience of procrastination after using the app. The participants were mainly high schoolers who have all experienced procrastination at some point in their high school career. This is shown clearly during the initial interview as a majority of the participants reported to have a tendency to procrastinate when doing assignments for school or studying for exams. However, when we compared the participants' post-experience with procrastination to their initial experience, the result was overall very positive: out of the 20 participants, 16 of them reported that they are less likely to procrastinate when they have entered the task to the small goal list in the app, while 4 of them reported no changes to their experience with procrastination, which suggests that a majority of the user base tend to benefit from app in terms of reducing procrastination.

We then collected evaluations from the participants by asking them to list out the things that they liked and/or disliked about the app. Some things that the participants liked about the app were features such as the rank system, the clear display of small goals along with the notification system, and the message that you can write to yourself when entering each goal. Some participants reported that the rank system really motivated them to finish the assignments that they set off to do since they could all see each other on the leaderboard and wanted to have a higher rank than the other participants. The clean display of small goals was also a favorable feature as many of the participants reported that they could easily create and manage a schedule using the app. Some things that people disliked about the app were features like the restriction of having only one big goal at a time and the predefined goals being too limited. Some of the participants reported that the app only allows them to create one big goal at a time, which really limits their aspiration to complete more goals. Some also complained that the list of predefined goals were far too limited and suggested more variations of small goals should be added to the 
list. However, the dissatisfaction with other functions of the app was still very minimal, suggesting that the app was overall a very effective tool to help reduce procrastination.

Lastly, we asked the participants to give any feedback or suggestions for the app in terms of improvements of certain parts and the implementation of other features in the future. One of the most important feedback that we received was that the social feature of the app can be further expanded to include other forms of social interactions between the users. For example, some of the participants felt that it would be more exciting if they could make friends with each other and check on each other's progress by chatting with them while using the app. Moreover, some people also suggested that we should implement a calendar system as a better form of visualization for the user's schedule. We believe that all of these suggestions are extremely valuable to the future improvements of the app, and we are currently planning on implementing these features while addressing the complaints by making changes to some of the existing parts of the app.

\section{Related Work}

Forest is a popular productivity app made by SEEKRTECH CO., LTD. that helps people manage their time and stay focused on the tasks at hand by letting them plant trees in the app when they need to complete a task for a certain time without being distracted by the phone [10]. Similar to iProgress, Forest also has a reward system in which users can spend the coins they obtained to unlock new types of trees and bushes, or even plant real trees on Earth. This is an incredibly interesting feature as it creates a sense of responsibility and achievement for the users as they can protect the environment while becoming more productive. However, unlike iProgress, Forest does not have a rank feature or a customizable schedule.

Another related app is Momentum Habit Tracker developed by Mathias Maehlum [9]. Momentum is an ideal app for keeping track of habits and routines as it offers a calendar feature that allows users to create weekly targets and take notes on their progress, making it very easy to build new habits. Although the app is similar to iProgress in that they both let users create goals and keep track of them, Momentum is more like a calendar that helps people with developing new habits, while iProgress focuses more on addressing the problem of procrastination by providing incentive.

Habitica is another interesting app that helps people track and maintain good habits [8]. Similar to iProgress, Habitica also has a creative way to give people extra incentive to stay motivated. Inspired by RPG video games, the users of Habitica can rank up their characters as they complete more tasks, which makes habit building more fun and exciting. Compared to Habitica, iProgress does not have a complex RPG game reward system, but it does have a more goal oriented system that targets the emotions of the users, making it more effective in helping people beat procrastination.

\section{Conclusion And Future Work}

To summarize, procrastination is a common issue that many people face today, and it can often have many negative effects on a person such as increased guilt and frustration, poor performance at work or school, and lower productivity. While most people do not experience the most severe consequences of procrastination, this issue is still prevalent as it can often limit the success of those who do procrastinate. Therefore, in this project, our goal is to address the problem of procrastination by creating a mobile application that combines all the tools and strategies that are effective dealing with this issue. With unique features such as a rank system that will motivate 
users to complete more goals without procrastinating by creating a competitive environment, and a customizable message attached to each goal that allows the users to consciously note the importance of avoiding procrastination, iProgress can help people to effectively deal with the problem of procrastination and form a better and more productive lifestyle by developing good work ethics and more healthy habits. Through the evaluations and feedback from some of the users, we were able to determine that the app has an overall positive effect on people who struggle with procrastination. We found that the majority of people who use the app tend to benefit from it in terms of reducing their tendency to procrastinate. As for the app's limitation, there are definitely a few changes that need to be made in order to improve the overall user experience. One of the limitations is that iProgress's social feature is not very comprehensive as it does not allow users to interact with each other in the forms of chatting or making friends. However, we are currently planning on expanding the social feature in the near future. The first step would be to add a message system where the users can leave each other messages as reminders of the goals that they need to complete. Other changes and features that we heard from user suggestions are also going to be implemented. For example, we will add a calendar page to the app so that the user can create and manage schedules more easily. We will also expand the restriction on the number of big goals so that people can have multiple big goals at a time. In the meantime, we will continue to collect feedback and suggestions from users and make changes to the app so that more people will benefit from it by being more productive and freer of procrastination.

\section{REFERENCES}

[1] Klingsieck, Katrin B. "Procrastination." European Psychologist (2013).

[2] Solomon, Laura J., and Esther D. Rothblum. "Academic procrastination: Frequency and cognitivebehavioral correlates." Journal of counseling psychology 31.4 (1984): 503.

[3] Lay, Clarry H. "At last, my research article on procrastination." Journal of research in personality 20.4 (1986): 474-495.

[4] Mazur, James E. "Procrastination by pigeons: Preference for larger, more delayed work requirements." Journal of the Experimental Analysis of Behavior 65.1 (1996): 159-171.

[5] Lay, Clarry H., and Henri C. Schouwenburg. "Trait Procrastination, Time Management." Journal of social Behavior and personality 8.4 (1993): 647-662.

[6] Kuzmin, Nikita, Konstantin Ignatiev, and Denis Grafov. "Experience of Developing a Mobile Application Using Flutter." Information Science and Applications. Springer, Singapore, 2020. 571575.

[7] Moroney, Laurence. "The firebase realtime database." The Definitive Guide to Firebase. Apress, Berkeley, CA, 2017. 51-71.

[8] Habitica: Gamified Taskmanager. HabitRPG, Inc,2020. Vers.2.7. Google Play Store. https://play.google.com/store/apps/details?id=com.habitrpg.android.habitica\&hl=en_US

[9] Mathias Maehlum. Momentum Habit Tracker - Routines, Goals \& Rituals. Momentum.cc, 2020. Vers. 3.5. Apple App Store. https://apps.apple.com/us/app/momentum-habit-tracker-routinesgoalsrituals/id946923599

[10] Forest: Stay focused. SEEKRTECH CO., LTD,2020. Vers.4.20.0. Google Play Store. https://play.google.com/store/apps/details?id=cc.forestapp\&hl=en_US

[11] Paden, Nita, and Roxanne Stell. "Reducing procrastination through assignment and course design." Marketing Education Review 7.2 (1997): 17-25.

[12] Lamwers, Linda L., and Christine H. Jazwinski. "A comparison of three strategies to reduce student procrastination in PSI." Teaching of Psychology 16.1 (1989): 8-12.

[13] Tice, Dianne M., and Roy F. Baumeister. "Longitudinal study of procrastination, performance, stress, and health: The costs and benefits of dawdling." Psychological science 8.6 (1997): 454-458.

(C) 2020 By AIRCC Publishing Corporation. This article is published under the Creative Commons Attribution (CC BY) license. 